Annals of Pure and Applied Mathematics

Vol. 21, No. 2, 2020, 95-102

ISSN: 2279-087X (P), 2279-0888(online)

Published on 21 April 2020

Annals of

www.researchmathsci.org

DOI: http://dx.doi.org/10.22457/apam.v21n2a3654

\title{
Stabilizer Graph and Orbit Graph of Finite Groups
}

\author{
K. Annadurai ${ }^{1}$ and M. Bhuvaneshwari ${ }^{2 *}$
}

PG and Research Department of Mathematics, M.V. Muthiah Government Arts College for Women, Mother Teresa Women's University, Dindigul - 624 001, India.

11Email: drkannadurai@gmail.com

*Corresponding author. ${ }^{2}$ Email: bhuvanika1524@ gmail.com

Received 17 February 2020; accepted 29 March 2020

Abstract. The interplay between groups and graphs have been the most famous and productive area of algebraic graph theory. In this paper, we define new graphs namely stabilizer graph, orbit graph, non-stabilizer graph and non-orbit graph for each permutation group G. Our aim is to translate properties of graphs into algebraic properties and then using the results and methods of algebra to declare theorem about graphs. We investigate the interconnection between the group theoretic properties of $\mathrm{G}$ and the graph theoretic properties of the above said graphs. Some of the graphs are illustrated with example. Finally we obtain some important results including theorem and discuss the relationship between group isomorphism and graph isomorphism.

Keywords: Permutation Group; Stabilizer of a point; Orbit of a point; Group isomorphism; Graph isomorphism.

\section{AMS Mathematics Subject Classification (2010): 82D25}

\section{Introduction}

Graph theory is one of the foremost probing field in mathematics principally because of its applications in various fields which include biochemistry, electrical engineering, computer science and operations research. These both twigs of mathematics are playing a prominent role in modern mathematics.

Groups are the focal mathematical equipment for studying symmetries of an object and symmetries are usually associated to graph automorphisms. Several structures in abstract algebra are special cases of groups. In group theory we study and examine different groups and their structures while in graph theory we concentrate on the graphs that indicates the structure of materials and objects. The forceful combinatorial methods found in graph theory have also been utilized to demonstrate significant and well-known results in a variety of areas in mathematics comprising group theory.

All finite groups can be delineated as the automorphism group of a connected graph [1]. Relating a graph to a group provides a method of visualizing a group and connects two important branches of mathematics. It gives a review of cyclic groups, dihedral groups, direct products, generators and relations [2]. In the past few decades the researchers have concentrated on the modified form of groups and graphs by inter relating their properties. The literature of algebraic graph theory itself has grown 
K. Annadurai and M. Bhuvaneshwari

enormously since 1974 [3]. The study of the algebraic structures utilizing the properties of graphs has become a rousing research topic in the last twenty years, foremost to several interesting results and questions.

The concept of zero-divisor graph was first introduced by Beck in [4] while discussing the coloring of a commutative ring. In his work all elements of the ring were considered as vertices of the graph. Anderson and Naseer used this same concept in [5] and Mahadevi and Babujee also done their work on special structures of zero-divisor graphs [6]. Muneshwar and Bondar gave an introduction and overview of some nice ideas from group theory by using graph theory [7]. Chalapathi and Kiran Kumar explained the concept of order divisor graph of finite groups [8]. Cameron and Ghosh done their work on power graph of a finite group [9]. Omer, Sarmin and Erfanian introduced orbit graph for some finite solvable groups [10]. Shigehalli and Uppin discussed some properties of glue graph [11]. Varkey and Rajan studied on the spectrum and energy of concatenated singular graphs [12]. Revankar et al. [13] explained on eccentricity sum eigenvalue and eccentricity sum energy of a graph. In this paper, we construct a stabilizer graph and orbit graph for finite groups.

In section 2, we discuss stabilizer graph and non-stabilizer graph. Orbit graph and non-orbit graph are discussed in section 3. In each section related theorems and results are obtained. Also, some of graphs are illustrated with suitable examples. Section 4 concludes the paper.

\section{Stabilizer graph of finite group}

A. Stabilizer graph of $\mathbf{G}$

Definition 2.1. Let $\mathrm{G}$ be a group of permutations of a set A. For each $\mathrm{x}$ in $\mathrm{A}$, let $\operatorname{Stab}_{G}(x)=\{\phi \in \mathrm{G} \mid \phi(\mathrm{x})=\mathrm{x}\}$. We call $\operatorname{Stab}_{G}(x)$ the stabilizer of $\mathrm{x}$ in $\mathrm{G}$ and is denoted by $S_{G}(x)$. (See [14]).

Definition 2.2. Let $\mathrm{A}$ be any finite set and $\mathrm{G}$ be a group of permutations. We define a stabilizer graph of $\mathrm{G}$, denoted by $\Gamma\left(S_{G}(A)\right)$ is a simple graph $(\mathrm{V}, \mathrm{E})$ with vertex set $\mathrm{V}=\{$ $\left.S_{G}(x) \mid \forall \mathrm{x} \in \mathrm{A}\right\}$ and edge set $\mathrm{E}=\left\{\left(S_{G}(x), S_{G}(y)\right) \mid S_{G}(x)=S_{G}(y) \forall \mathrm{x}, \mathrm{y} \in \mathrm{A}\right\}$.

Example 2.1. Let $A=U(7)=\{1,2,3,4,5,6\}$. Let us calculate the left rectangular representation $\overline{U(7)}$ for $\mathrm{U}(7)=\{1,2,3,4,5,6\}$. Writing the permutations of $\mathrm{U}(7)$ in array form, we have ( $T_{x}$ is just a multiplication by $\left.\mathrm{x}\right)$,

$$
\begin{array}{lll}
\mathrm{T}_{1}=\left(\begin{array}{llllll}
1 & 2 & 3 & 4 & 5 & 6 \\
1 & 2 & 3 & 4 & 5 & 6
\end{array}\right), & \mathrm{T}_{2}=\left(\begin{array}{llllll}
1 & 2 & 3 & 4 & 5 & 6 \\
2 & 4 & 6 & 1 & 3 & 5
\end{array}\right), \\
\mathrm{T}_{3}=\left(\begin{array}{llllll}
1 & 2 & 3 & 4 & 5 & 6 \\
3 & 6 & 2 & 5 & 1 & 4
\end{array}\right), & \mathrm{T}_{4}=\left(\begin{array}{llllll}
1 & 2 & 3 & 4 & 5 & 6 \\
4 & 1 & 5 & 2 & 6 & 3
\end{array}\right), \\
\mathrm{T}_{5}=\left(\begin{array}{llllll}
1 & 2 & 3 & 4 & 5 & 6 \\
5 & 3 & 1 & 6 & 4 & 2
\end{array}\right), & \mathrm{T}_{6}=\left(\begin{array}{llllll}
1 & 2 & 3 & 4 & 5 & 6 \\
6 & 5 & 4 & 3 & 2 & 1
\end{array}\right) .
\end{array}
$$

Let $\mathrm{G}=\overline{U(7)}=\{(1),(124)(365),(132645),(142)(356),(15462$ 3),(1 6)(2 5)(3 4)\}. Then, we have, 
$\operatorname{Stab}_{G}(1)=\operatorname{Stab}_{G}(2)=\operatorname{Stab}_{G}(3)=\operatorname{Stab}_{G}(4)=\operatorname{Stab}_{G}(5)=\operatorname{Stab}_{G}(6)=\{(1)\} \cdot$ Stabilizer graph of $U(7)$ in $\frac{G(7)}{U(s ~ s h o w n ~ i n ~ F i g u r e ~} 1$.

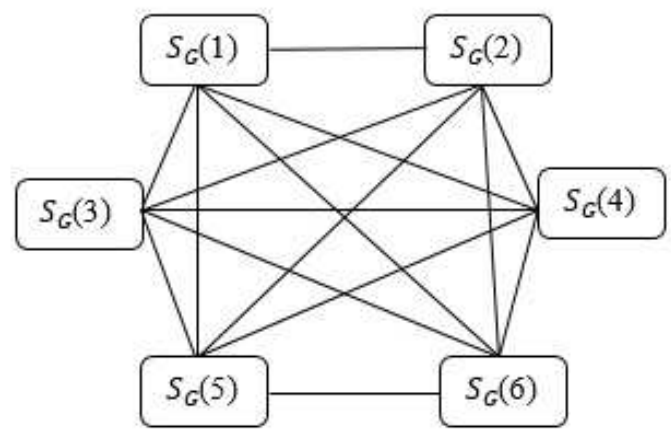

Figure 1: $\Gamma\left(\mathrm{S}_{\overline{\mathrm{U}(7)}} \mathrm{U}(7)\right)$

Definition 2.3. $\Gamma\left(S_{G}(A)\right)$ is complete if and only if $S_{G}(x)=S_{G}(y)$ for all distinct $\mathrm{x}, \mathrm{y} \in \mathrm{A}$.

Result 2.1. Stabilizer graph of the group $\overline{U(n)}$ is always complete.Because the stabilizer point of $\mathrm{U}(\mathrm{n})$ in $\overline{U(n)}$ is trivially equal to (1).

Result 2.2. If $\mathrm{G}$ is a symmetric group $S_{n}$ where $\mathrm{n} \geq 3$, then the stabilizer graph is always null graph.

Proof: Let A be any finite set and $\mathrm{G}$ be a group of permutations of a set A. For each $\mathrm{x}$ in $\mathrm{A}$, let $\operatorname{Stab}_{G}(x)=\{\phi \in G \mid \phi(x)=x\}$. If $\mathrm{G}$ is a symmetric group $S_{n}$ where $n \geq 3$, we get different stabilizer of each $\mathrm{x}$ in G. i.e. $S_{G}(x) \neq S_{G}(y) \forall x, y \in A$. Then there does not exist an edge between every pair of vertices, $S_{G}(x) \forall x \in A$. Therefore, the stabilizer graph $\Gamma\left(S_{G}(A)\right)$ is a null graph.

Result 2.3. For abelian groups, the stabilizer graph is obviously complete.

Proof: Let $\mathrm{G}$ be an abelian group. i.e., $S_{n}, n \leq 2$ and $\overline{U(n)}$. For each $\mathrm{x}$ in $\mathrm{A}$, we have $\operatorname{Stab}_{G}(x)=\{(1)\}$ so that $S_{G}(x)=S_{G}(y) \forall x, y \in A$. Then there exist an edge between every pair of vertices, $S_{G}(x) \forall x \in A$. Therefore, the stabilizer graph $\Gamma\left(S_{G}(A)\right)$ is complete.

Result 2.4. If $G$ is the set of all even permutations of a symmetric group, then the stabilizer graph is complete for $S_{n}$ where $\mathrm{n} \leq 3$ and null graph for $S_{n}$ where $\mathrm{n} \geq 4$.

Proof: Let G be the set of all even permutations of a symmetric group $S_{n}$.

Case(i): If $\mathrm{G}$ is the set of all even permutations of a symmetric group $S_{n}$ where $n \leq 3$, then for each $\mathrm{x}$ in A, we have $\operatorname{Stab}_{G}(x)=\{(1)\}$ so that $S_{G}(x)=S_{G}(y) \forall x, y \in A$. Then there exist an edge between every pair of vertices, $S_{G}(x) \forall x \in A$. Therefore, the stabilizer graph $\Gamma\left(S_{G}(A)\right)$ is complete.

Case(ii): If $\mathrm{G}$ is the set of all even permutations of a symmetric group $S_{n}$ where $n \geq 4$, then for each $\mathrm{x}$ in $\mathrm{A}, S_{G}(x) \neq S_{G}(y)$ for all $x, y \in A$. Then there does not exist an edge between every pair of vertices, $S_{G}(x)$ for all $x \in A$. Therefore, the stabilizer graph $\Gamma\left(S_{G}(A)\right)$ is a null graph. 
Result 2.5. If $\mathrm{G}$ is the set of all odd permutations of a symmetric group, then the stabilizer graph is null graph for $S_{n}$ where $\mathrm{n} \geq 3$ and empty graph for $S_{2}$.

Proof: Let $\mathrm{G}$ be the set of all odd permutations of a symmetric group $S_{n}$.

Case (i): If $\mathrm{G}$ is the set of all odd permutations of a symmetric group $S_{n}$ where $n \geq$ 3 , then for each $\mathrm{x}$ in $\mathrm{A}, S_{G}(x) \neq S_{G}(y) \forall x, y \in A$. Then there does not exist an edge between every pair of vertices, $S_{G}(x) \forall x \in A$. Therefore, the stabilizer graph $\Gamma\left(S_{G}(A)\right)$ is a null graph.

Case (ii): If $\mathrm{G}$ is the set of all odd permutations of a symmetric group $S_{n}$ where $n=2$, then for each $\mathrm{x}$ in $\mathrm{A}$, stabilizer point does not exist. So there is no any vertex. Thus we get an empty graph.

Result 2.6. If $\mathrm{G}$ is the set of all even permutations of a group $\overline{U(n)}$, then the stabilizer graph is complete.

Proof: Let $\mathrm{G}$ be a group of even permutations of the group $\overline{U(n)}$. Then, by Result 2.1, for each $\mathrm{x}$ in $\mathrm{A}, \operatorname{Stab}_{G}(x)=\{(1)\}$ so that $S_{G}(x)=S_{G}(y) \forall x, y \in A$. Then there exist an edge between every pair of vertices, $S_{G}(x) \forall x \in A$. Therefore, the stabilizer graph $\Gamma\left(S_{G}(A)\right)$ is complete.

Result 2.7. If $\mathrm{G}$ is the set of all odd permutations of a group $\overline{U(n)}$, then the stabilizer graph is empty graph.

Example 2.2. Let $\mathrm{A}=\mathrm{U}(5)=\{1,2,3,4,5\}$. If we take $\mathrm{G}$ as an odd permutation then $\mathrm{G}=$ $\left.\overline{U(5)}=\left\{\begin{array}{llll}1 & 2 & 4 & 3\end{array}\right),\left(\begin{array}{llll}1 & 3 & 4 & 2\end{array}\right)\right\}$.

Result 2.8. If we take $G$ as a random permutations of a symmetric group, then the stabilizer graph maybe connected or disconnected.

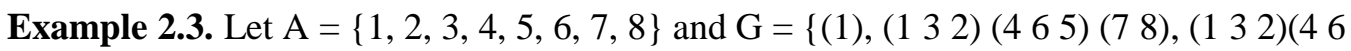
5), (1 23 ) (4 5 6), (1 23 ) (4 5 6) (7 8), (7 8) \}. Then, we have,

$\operatorname{Stab}_{G}(1)=\operatorname{Stab}_{G}(2)=\operatorname{Stab}_{G}(3)=\operatorname{Stab}_{G}(4)=\operatorname{Stab}_{G}(5)=\operatorname{Stab}_{G}(6)=\{(1),(78)\}$,

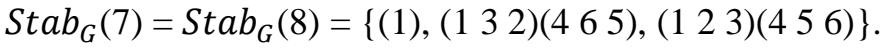

Theorem 2.1. Let $\mathrm{U}\left(n_{1}\right)$ and $\mathrm{U}\left(n_{2}\right)$ be two finite groups. If $\mathrm{U}\left(n_{1}\right) \cong \mathrm{U}\left(n_{2}\right)$, then $\Gamma\left(S_{\overline{U\left(n_{1}\right)}} U\left(n_{1}\right)\right) \cong \Gamma\left(S_{\overline{U\left(n_{2}\right)}} U\left(n_{2}\right)\right)$.

Proof: Assume $\mathrm{U}\left(n_{1}\right) \cong \mathrm{U}\left(n_{2}\right)$. Then there exist an isomorphic map $\phi: \mathrm{U}\left(n_{1}\right) \rightarrow \mathrm{U}\left(n_{2}\right)$. Since $\phi$ is a group isomorphism that preserves stabilizer point of every element of a set, $\phi$ maps stabilizer point of $\mathrm{U}\left(n_{1}\right)$ to a stabilizer point of $\mathrm{U}\left(n_{2}\right)$. Let $x_{i}$ and $y_{i}$ be the stabilizer points of $\mathrm{U}\left(n_{1}\right)$ and $\mathrm{U}\left(n_{2}\right)$ respectively, where $\mathrm{i}=1,2,3 \ldots \mathrm{n}$. Then we see that $\phi\left(x_{i}\right)=y_{i}$ for all i. Also, if $x_{i}$ is adjacent to $x_{j}$, then $\phi\left(x_{i}\right)$ is adjacent to $\phi\left(x_{j}\right)$, for all $i$ $\neq \mathrm{j}$. Thus $\phi$ is a graph isomorphism. Hence $\Gamma\left(S_{\overline{U\left(n_{1}\right)}} U\left(n_{1}\right)\right) \cong \Gamma\left(S_{\overline{U\left(n_{2}\right)}} U\left(n_{2}\right)\right) \square$ 


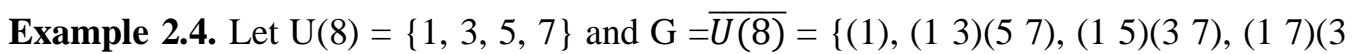
5)\}. Then, we have $\operatorname{Stab}_{G}(1)=\operatorname{Stab}_{G}(3)=\operatorname{Stab}_{G}(5)=\operatorname{Stab}_{G}(7)=\{(1)\}$. Similarly, let $\mathrm{U}(12)=\{1,5,7,11\}$ and $\mathrm{G}=\overline{U(12)}=\{(1),(15)(711),(17)(511),(111)(5$ 7) $\}$. Then, we have,

$\operatorname{Stab}_{G}(1)=\operatorname{Stab}_{G}(5)=\operatorname{Stab}_{G}(7)=\operatorname{Stab}_{G}(11)=\{(1)\}$. Stabilizer graph of U(8) in $\overline{U(8)}$ and Stabilizer graph of $\mathrm{U}(12)$ in $\overline{U(12)}$ is shown in Figure 2.
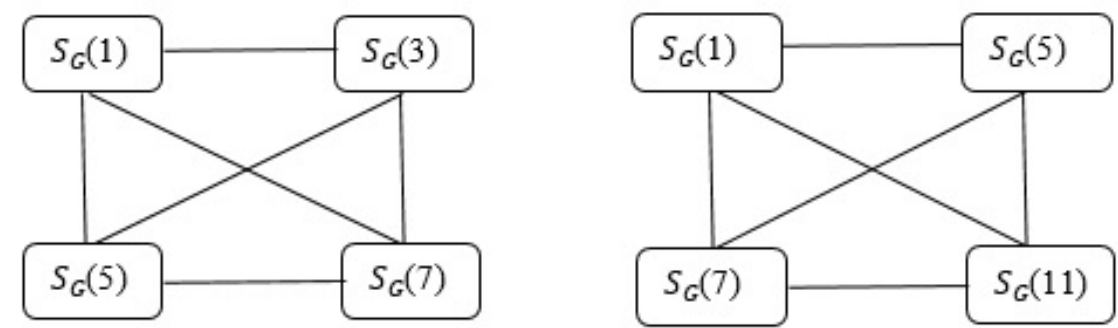

Figure 2: Graph isomorphism between $\Gamma\left(\mathrm{S}_{\overline{\mathrm{U}(8)}}(\mathrm{U}(8))\right)$ and $\Gamma\left(\mathrm{S}_{\overline{\mathrm{U}(12)}}(\mathrm{U}(12))\right)$.

Corollary 2.1. The converse of the above theorem is not true.

Proof: Consider the finite groups $\mathrm{U}(8)$ and $\mathrm{U}(10)$. Let $\mathrm{U}(8)=\{1,3,5,7\}$ and $\mathrm{G}=\overline{U(8)}$ $=\left\{(1),\left(\begin{array}{ll}1 & 3\end{array}\right)(5\right.$ 7) $),\left(\begin{array}{ll}1 & 5\end{array}\right)(3$ 7) $),\left(\begin{array}{l}1 \\ \text { 7 }\end{array}\right)(3$ 5) $\}$ and Let $\mathrm{U}(10)=\{1,3,7,9\}$ and $\mathrm{G}=\overline{U(10)}$

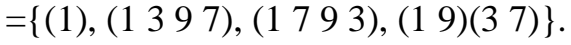

Then stabilizer point of $\mathrm{U}(8)$ in $\overline{U(8)}$ and stabilizer point of $\mathrm{U}(10)$ in $\overline{U(10)}$ are $\operatorname{Stab}_{G}(1)=\operatorname{Stab}_{G}(3)=\operatorname{Stab}_{G}(5)=\operatorname{Stab}_{G}(7)=\{(1)\}$ and $\operatorname{Stab}_{G}(1)=\operatorname{Stab}_{G}(3)=$ $\operatorname{Stab}_{G}(7)=\operatorname{Stab}_{G}(9)=\{(1)\}$ respectively.

Here $\Gamma\left(S_{\overline{U(8)}}(U(8))\right)$ and $\Gamma\left(S_{\overline{U(10)}}(U(10))\right)$ are isomorphic, but $\mathrm{U}(8)$ and $\mathrm{U}(10)$ are not isomorphic. Stabilizer graph of $\mathrm{U}(8)$ in $\overline{U(8)}$ and Stabilizer graph of $\mathrm{U}(10)$ in $\overline{U(10)}$ is shown in Figure 3.
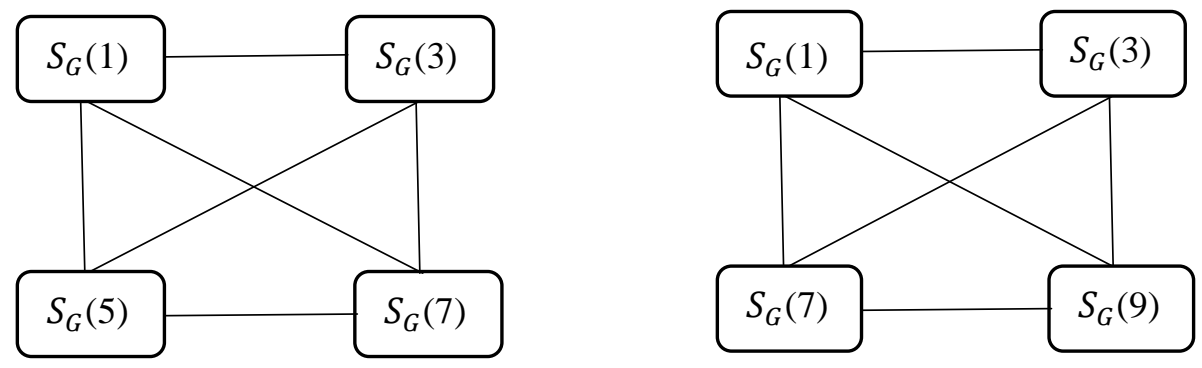

Figure 3: Graph isomorphism between $\Gamma\left(\mathrm{S}_{\overline{\mathrm{U}(8)}}(\mathrm{U}(8))\right)$ and $\Gamma\left(\mathrm{S}_{\overline{\mathrm{U}(10)}}(\mathrm{U}(10))\right)$.

\section{B. Non-stabilizer graph of $\mathbf{G}$}

Definition 2.4. Let $\mathrm{A}$ be any finite set and $\mathrm{G}$ be a group of permutations. We define a non-stabilizer graph of $\mathrm{G}$, denoted by $\Gamma_{N}\left(S_{G}(A)\right)$ is a simple graph $(\mathrm{V}, \mathrm{E})$ with vertex set $\mathrm{V}=\left\{S_{G}(x) \mid \forall \mathrm{x} \in \mathrm{A}\right\}$ and edge set $\mathrm{E}=\left\{\left(\left(S_{G}(x), S_{G}(y)\right) \mid S_{G}(x) \neq S_{G}(y) \forall \mathrm{x}, \mathrm{y} \in \mathrm{A}\right\}\right.$.

Note: Null graph and disconnected graph can be converted into a connected graph by using the non-stabilizer graph definition. 
K. Annadurai and M. Bhuvaneshwari

Result 2.9. If $\mathrm{G}$ is a symmetric group $S_{n}$ where $\mathrm{n} \geq 3$, then the non-stabilizer graph is always complete.

Proof: Let $\mathrm{G}$ be a symmetric group $S_{n}$ where $n \geq 3$. Then, for each $\mathrm{x}$ in $\mathrm{A}, S_{G}(x) \neq$ $S_{G}(y) \forall x, y \in A$. By the definition of non-stabilizer graph, there exist an edge between every pair of vertices, $S_{G}(x) \forall x \in A$. Therefore, the non-stabilizer graph $\Gamma_{N}\left(S_{G}(A)\right)$ is complete.

Result 2.10. Non-stabilizer graph need not be complete.

Note: Disconnected graph becomes connected when using non stabilizer graph definition but this need not be complete.

\section{Orbit graph of finite group}

\section{A. Orbit graph of $\mathbf{G}$}

Definition 3.1. Let $\mathrm{G}$ be a group of permutations of a set $\mathrm{A}$. For each $\mathrm{x}$ in $\mathrm{A}$, let $O r b_{G}(x)$ $=\{\phi(\mathrm{x}) \mid \phi \in \mathrm{G}\}$. Then the set $\operatorname{Orb}_{G}(x)$ is a subset of A called the orbit of $\mathrm{x}$ under $\mathrm{G}$ and is denoted by $O_{G}(x)$. We use $\left|\operatorname{Orb}_{G}(x)\right|$ to denote the number of elements in $\operatorname{Orb}_{G}(x)$. (See [14]).

Definition 3.2. Let $\mathrm{A}$ be any finite set and $\mathrm{G}$ be a group of permutations. We define a orbit graph of $\mathrm{G}$, denoted by $\Gamma\left(O_{G}(A)\right)$ is a simple graph $(\mathrm{V}, \mathrm{E})$ with vertex set $\mathrm{V}=\{$ $\left.O_{G}(x) \mid \forall \mathrm{x} \in \mathrm{A}\right\}$ and edge set $\mathrm{E}=\left\{\left(O_{G}(x), O_{G}(y)\right) \mid O_{G}(x)=O_{G}(y) \forall \mathrm{x}, \mathrm{y} \in \mathrm{A}\right\}$.

Example 3.1. Let $\mathrm{A}=\{1,2,3\}$ and $\mathrm{G}=S_{3}=\left\{\left(\begin{array}{l}1\end{array}\right),\left(\begin{array}{lll}1 & 2 & 3\end{array}\right),\left(\begin{array}{lll}1 & 3 & 2\end{array}\right),\left(\begin{array}{ll}2 & 3\end{array}\right),\left(\begin{array}{ll}1 & 3\end{array}\right),\left(\begin{array}{ll}1 & 2\end{array}\right)\right\}$. Then, $\operatorname{Orb}_{G}(1)=\operatorname{Orb}_{G}(2)=\operatorname{Orb}_{G}(3)=\{1,2,3\}$. The Orbit graph of A under $S_{3}$ is shown in Figure 4.

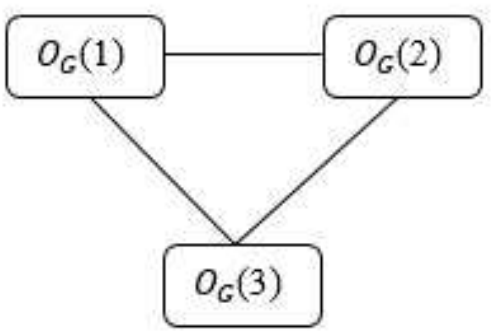

Figure 4: $\Gamma\left(\mathrm{O}_{\mathrm{S}_{3}}(\mathrm{~A})\right)$

Definition 3.3. $\Gamma\left(O_{G}(A)\right)$ is complete if and only if $O_{G}(\mathrm{x})=O_{G}(\mathrm{y})$ for all distinct $\mathrm{x}, \mathrm{y} \in \mathrm{A}$.

Result 3.1. If $\mathrm{G}$ is a symmetric group, then the orbit graph is always complete.

Proof: Let $\mathrm{A}$ be any finite set and $\mathrm{G}$ be a group of permutations of a set $\mathrm{A}$. For each $\mathrm{x}$ in A, let $\operatorname{Orb}_{G}(x)=\{\phi(x) \mid \phi \in G\}$. If $\mathrm{G}$ is a symmetric group, then for each $\mathrm{x}$ in A we get $O_{G}(x)=\{1,2,3, \ldots, \mathrm{n}\}$ so that $O_{G}(x)=O_{G}(y) \forall x, y \in A$. Then there exist an edge between every pair of vertices, $O_{G}(x) \forall x \in A$. Therefore, the Orbit graph $\Gamma\left(O_{G}(A)\right)$ is complete. 


\section{Stabilizer Graph and Orbit Graph of Finite Groups}

Result 3.2. Orbit graph of the group $\overline{U(n)}$ is always complete.

Proof: Let $\mathrm{G}$ be the group $\overline{U(n)}$. Then for each $\mathrm{x}$ in $\mathrm{A}$, we get $O_{G}(x)=\{1,2,3, \ldots, \mathrm{n}\}$ so that $O_{G}(x)=O_{G}(y) \forall x, y \in A$. Then there exist an edge between every pair of vertices, $O_{G}(x) \forall x \in A$. Therefore, the Orbit graph $\Gamma\left(O_{G}(A)\right)$ is complete.

Result 3.3. If $\mathrm{G}$ is the set of all even permutations of a symmetric group, then the orbit graph is always complete, except $S_{2}$.

Proof: Let $\mathrm{G}$ be the set of all even permutations of a symmetric group $S_{n}$.

Case (i): If $\mathrm{G}$ is the set of all even permutations of a symmetric group $S_{n}$ where $n \neq 2$, then for each $\mathrm{x}$ in $\mathrm{A}$, we get $O_{G}(x)=\{1,2,3, \ldots, \mathrm{n}\}$ so that $O_{G}(x)=O_{G}(y), \forall x, y \in A$. Then there exist an edge between every pair of vertices, $O_{G}(x) \forall x \in A$. Therefore, the Orbit graph $\Gamma\left(O_{G}(A)\right)$ is complete.

Case (ii): If $\mathrm{G}$ is the set of all even permutations of a symmetric group $S_{n}$ where $n=2$, then for each $\mathrm{x}$ in $\mathrm{A}$, we get different orbit points so that $O_{G}(x) \neq O_{G}(y) \forall x, y \in A$. Then there does not exist an edge between the pair of vertices, $O_{G}(x)$ and $O_{G}(y)$. Therefore, the Orbit graph $\Gamma\left(O_{G}(A)\right)$ is a null graph.

Result 3.4. If $\mathrm{G}$ is the set of all odd permutations of a symmetric group, then the orbit graph is always complete, except $S_{2}$.

Proof: Similar to the proof of Result 3.3.

Theorem 3.1. Let $\mathrm{U}\left(n_{1}\right)$ and $\mathrm{U}\left(n_{2}\right)$ be two finite groups. If $\mathrm{U}\left(n_{1}\right) \cong \mathrm{U}\left(n_{2}\right)$, then $\Gamma\left(O \overline{U\left(n_{1}\right)} U\left(n_{1}\right)\right) \cong \Gamma\left(O_{\overline{U\left(n_{2}\right)}} U\left(n_{2}\right)\right)$.

Proof: Similar to the proof of Theorem 2.1.

Corollary 3.1. The converse of the above theorem is not true.

Proof: Similar to the proof of Corollary 2.1.

\section{B. Non-orbit graph of $\mathbf{G}$}

Definition 3.4. Let $\mathrm{A}$ be any finite set and $\mathrm{G}$ be a group of permutations. We define a non-orbit graph of $\mathrm{G}$, denoted by $\Gamma_{N}\left(O_{G}(A)\right)$ is a simple graph $(\mathrm{V}, \mathrm{E})$ with vertex set $\mathrm{V}=$ $\left\{O_{G}(x) \mid \forall \mathrm{x} \in \mathrm{A}\right\}$ and edge set $\mathrm{E}=\left\{\left(O_{G}(x), O_{G}(y)\right) \mid O_{G}(x) \neq O_{G}(y), \forall \mathrm{x}, \mathrm{y} \in \mathrm{A}\right\}$.

Example 3.2. Let $A=\{1,2\}$ and $G=\left\{\left(\begin{array}{ll}1 & 2\end{array}\right)\right\}$. Then $\operatorname{Orb}_{G}(1)=\{2\}, \operatorname{Orb}_{G}(2)=\{1\}$ andnon-orbit graph of A under $\mathrm{G}$ is shown in Figure 5.

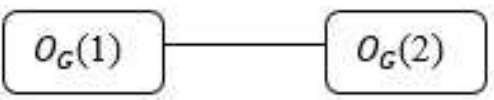

\section{Conclusion}

Figure 5: $\Gamma_{N}\left(\mathrm{O}_{\mathrm{G}}(\mathrm{A})\right)$

In this paper, we established the stabilizer graph and orbit graph of finite groups and some of the related results were dealt with suitable examples. Next, non-stabilizer graph and non-orbit graph were found and discussed. We investigated the interconnection between the group theoretic properties of $\mathrm{G}$ and the graph theoretic properties of the 
K. Annadurai and M. Bhuvaneshwari

stabilizer graph, orbit graph, non-stabilizer graph and non-orbit graph. This proposed work can be extended to other graph theoretic properties with other finite groups.

Acknowledgement. The authors are highly grateful to the anonymous reviewers for their valuable comments for improvement of the paper.

\section{REFERENCES}

1. R.Frucht, Graphs of degree three with a given abstract group, Canadian Journal of Mathematics, 1(4) (1949) 365-378.

2. S.U.Rehman, A.Q.Baig, M.Imran and Z.U.Khan, Order divisor graphs of finite groups, Analele Stiintifice ale Universitatii Ovidius Constanta, Seria Matematica, 26(3) (2018) 29-40.

3. N.Biggs, Algebraic Graph Theory, Cambridge university press, Cambridge, 1993.

4. I.Beck, Coloring of commutative rings, Journal of Algebra, 116(1) (1988) 208-226.

5. D.D.Anderson and M.Naseer, Beck's coloring of a commutative ring, Journal of Algebra, 159(2) (1993) 500-514.

6. P.Mahadevi and J.Baskar Babujee, On special structures of zero-divisor graphs, International Journal of Pure and Applied Mathematics, 119(13) (2018) 281-287.

7. R.A.Muneshwar and K.L.Bonder, On graph of a finite group, IOSR Journal of Mathematics, 10(5) (2014) 05-09.

8. T.Chalapathi and RVMSS Kiran Kumar, Order divisor graph of finite groups, Malaya Journal of Mathematik, 5(2) (2017) 464-474.

9. P.J.Cameron and S.Ghosh, The power graph of a finite group, Discrete Mathematics, 311(13) (2011) 1220-1222.

10. S.M.S.Omer, N.H.Sarmin and A.Erfanian, The orbit graph for some finite solvable groups, AIP Conference Proceedings, (2014) 863-869.

11. V.S.Shigehalli and G.V.Uppin, Some properties of glue graph, Annals of Pure and Applied Mathematics, 6(1) (2014) 98-103.

12. T.K.Mathew Varkey and John K. Rajan, On the spectrum and energy of concatenated singular graphs, Annals of Pure and Applied Mathematics, 14(3) (2017) 555-579.

13. D.S.Revankar, M.M.Patil and H.S.Ramane, On eccentricity sum eigenvalue and eccentricity sum energy of a graph, Annals of Pure and Applied Mathematics, 13(1) (2017) 125-130.

14. J.A.Gallian, Contemporary Abstract Algebra, CENGAGE Learning, $8^{\text {th }}$ Edition, 2012. 